\title{
Erarbeitung der Grundlagen für eine Prüfapparatur zur Bestimmung der Flammengeschwindigkeit in Staub/Luft-Gemischen
}

\author{
Katja Hüttenbrenner, Hannes Kern und Harald Raupenstrauch
}

Lehrstuhl für Thermoprozesstechnik, Montanuniversität Leoben, Leoben, Österreich

Eingegangen 5. Dezember 2019; angenommen 22. Januar 2020; online publiziert 10. Februar 2020

\begin{abstract}
Zusammenfassung: Damit die produzierende Industrie der Gefahr von Bränden und Explosionen entgegenwirken kann, ist neben vorbeugenden Maßnahmen zur Explosionsverhinderung die Auslegung von Sicherheitseinrichtungen essentiell. Ein Ansatz hierfür sind strömungsmechanische Berechnungsverfahren, mit deren Hilfe der Produktionsprozess berechnet werden kann und so genau auf diese spezifisch abgestimmten Sicherheitsmaßnahmen gesetzt werden können. Am Lehrstuhl für Thermoprozesstechnik an der Montanuniversität Leoben werden schon seit einiger Zeit im Bereich Modellbildung und Simulation Berechnungsmodelle entwickelt, um Staub- und Gasexplosionen auf Grundlage der sicherheitstechnischen Kenngrößen simulieren zu können. Diese Modelle müssen nach der Entwicklung mit Realwerten verglichen werden. Ziel der durchgeführten Arbeit war die Untersuchung der Flammenausbreitung in einem brennbaren Staub/Luft-Gemisch mit und ohne Inertstoffzumischung und die Betrachtung der durchgeführten Laborversuche mit berechneten Simulationsergebnissen dieser.
\end{abstract}

Schlüsselwörter: Staubexplosion,

Flammengeschwindigkeit, Explosionsunterdrückung, CFD-Simulation

Basic Research for the Development of a Novel Test Apparatus for the Measurement of Flame Velocities in Dust/air Mixtures

Abstract: In the process industries, most major accidents are still caused by fires and explosions. Actions and devices to mitigate and to prevent explosions are important and a consistent development is essential. In order to do

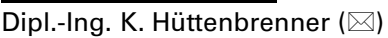

Lehrstuhl für Thermoprozesstechnik,

Montanuniversität Leoben,

Franz-Josef-Straße 18,

8700 Leoben, Österreich

katja.huettenbrenner@unileoben.ac.at so, fluid dynamic calculation methods are used. The Chair of Thermal Processing Technology of the Montanuniversitaet Leoben has been developing computational models for simulation purposes to calculate dust and gas explosions on the basis of safety parameters for some time. These models have to be compared with real data. The aim of the present work is the study of flame propagation in a mixture of combustible dust and air with and without inert material and the comparison of experimental results with calculation results.

Keywords: Dust explosion, Flame velocity, Explosion prevention, CFD simulation

\section{Einleitung}

Hohe Verluste in der produzierenden Industrie werden u. a. durch Brände und Explosionen hervorgerufen. Obwohl solche Vorkommnisse selten sind, besteht ein hohes Gefahrenpotential, da die Wahrscheinlichkeit von Anlagenverlust oder Todesfällen sehr hoch ist. Neben vorbeugenden Maßnahmen zur Explosionsverhinderung ist die korrekte Auslegung von Sicherheitseinrichtungen ein wesentlicher Bestandteil der Sicherheitsforschung auf dem Gebiet des Explosionsschutzes. Diese Maßnahmen dienen neben dem Schutz der Produktionsstätten vor allem dem Schutz der Menschen. Sowohl Arbeitnehmerlnnen als auch Nachbarn und andere Stakeholder des Betriebes sollen durch solche Einrichtungen im Anlassfall geschützt werden. Zur Auslegung von Sicherheitseinrichtungen ist die Kenntnis von sogenannten sicherheitstechnischen Kenngrößen von Bedeutung. Hierbei handelt es sich allerdings um keine physikalischen Größen, sondern um Kenngrößen, welche sich auf empirische Untersuchungen einzelner Stoffe und Zubereitungen stützen und daher vom Bestimmungsverfahren beeinflusst werden. Die Bestimmung der sicherheitstechnischen Kenngrößen erfolgt nach der Norm EN ISO 8007920-2 in Normapparaturen, u. a. der 20-Liter-Siwek-Appara- 


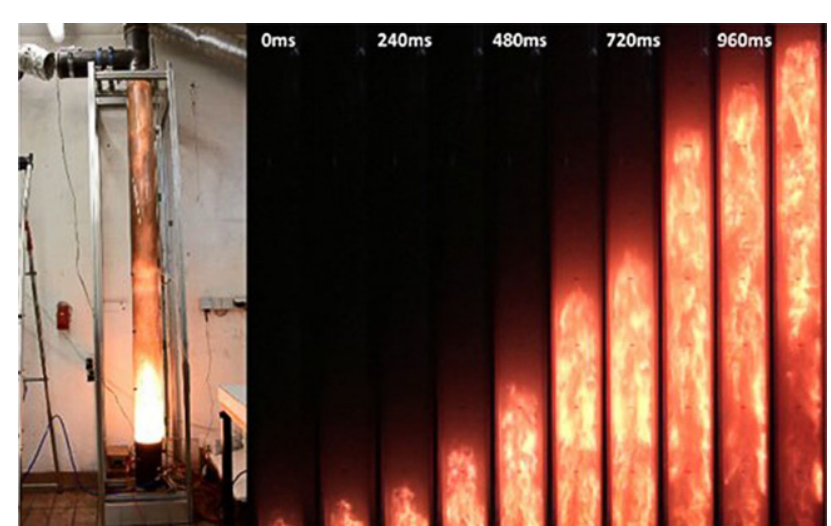

Abb. 1: Messung der Flammengeschwindigkeitin der Rohrapparatur am Lehrstuhl für Thermoprozesstechnik [4]

tur und der Apparatur zur Bestimmung der Mindestzündenergie, und bilden die Grundlage für die Auslegung und Konstruktion der Sicherheitseinrichtungen in der Industrie (z. B. Berstscheiben, Entkopplungseinrichtungen).

Probleme treten bei der Übertragbarkeit von sicherheitstechnischen Kenngrößen auf industrielle Anwendungen auf. "Bei Explosionsereignissen in der betrieblichen Praxis ist weder der momentane Turbulenzzustand noch der Zeitpunkt der Zündung bekannt" [1]. Um diesen Problemen aus dem Weg zu gehen, sind Alternativen zu den derzeit verwendeten Kenngrößen notwendig. Ein Ansatz ist die Verwendung von sogenannten "full CFD codes", also strömungsmechanische Berechnungsverfahren, mit deren Hilfe der Produktionsprozess im gefährdeten Anlagenteil über die Lösung der Erhaltungsgleichungen für Energie, Impuls usw. berechnet wird [2]. Ein Nachteil dieser Methode ist die Bestimmung der erforderlichen Stoffdaten für die Berechnung. Das größte Potential in der anlagenspezifischen Auslegung von Sicherheitseinrichtungen tragen die CFD-Methoden, wenn geeignete Kenngrößen gefunden werden, auf deren Basis die Berechnungen erfolgen können. Verschiedene wissenschaftliche Arbeiten zeigen, dass sich die laminare Flammengeschwindigkeit sehr gut als Grundlage dafür eignet [3,4]. Dieser Kennwert bringt den Vorteil, einen Parameter zu erhalten, der neben der Unabhängigkeit von Turbulenzbedingungen auch den Einfluss der Zündquelle stark reduziert [4]. (Abb. 1).

\section{Flammengeschwindigkeit}

Die Grundlagen für eine Prüfapparatur zur Bestimmung der Flammengeschwindigkeit wurden im von der FFG geförderten Projekt „TUBEX“ in Zusammenarbeit mit der Firma M.A.L. am Lehrstuhl für Thermoprozesstechnik erarbeitet. Nach der Zündung eines Staub/Luft-Gemisches erfolgt die Flammenfortpflanzung als Deflagration. Hierbei schreitet die Reaktionsfront mit einer Geschwindigkeit kleiner als die Schallgeschwindigkeit fort und die Druckfront bewegt sich mit Schallgeschwindigkeit von der Reaktionsfront weg [5]. Die Flammenfortpflanzungsgeschwindigkeit innerhalb der Reaktionsfront wird dabei von den auftretenden Wärme- und Stofftransportprozessen molekularer und turbulenter Art bestimmt [6]. Die Flammengeschwindigkeit eines Staub/Luft-Gemisches wird daher durch die Beschaffenheit des Brennstoff/Luft-Gemisches charakterisiert. Zur Bestimmung dieser wird im Allgemeinen die sogenannte TubeMethode angewendet, bei der der brennbare Staub in einer Rohrapparatur verteilt und an einem Ende gezündet wird. Die Flammenausbreitung sowie das Abbrandverhalten können so beobachtet und photometrisch erfasst werden. Im Vergleich zu einer sich ideall ausbreitenden Flammenfront zeigt sich diese in einer Rohrapparatur etwas verändert. Durch Wandeffekte und durch die Flammenausbreitung hervorgerufene Turbulenzerscheinungen nimmt die Front eine parabolische Form an [4]. Um Turbulenzeinflüsse bei den Versuchen so gering wie möglich zu halten, wurde ein Staubeintragsverfahren entwickelt, welches die Schwerkraft nutzt. Das Strömungsfeld in der Rohrapparatur wurde mithilfe der particle image velocimetry (PIV) Messung ermittelt (vgl. Abb. 2). Die Partikelbewegung wird dabei in
Abb. 2: Geschwindigkeitsfeld vor (a) und nach (b) der Zündung (Staubkonzentration zwischen 150 und $250 \mathrm{~g} / \mathrm{m}^{3}$ ) [7]
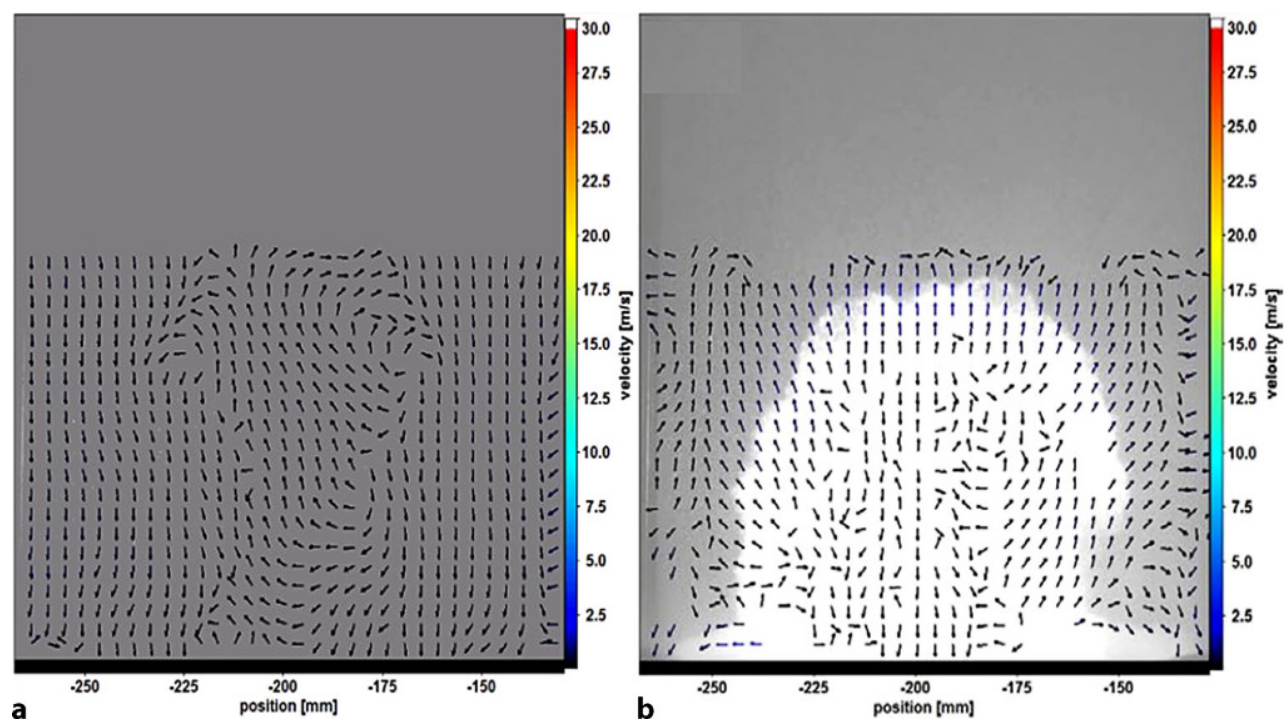

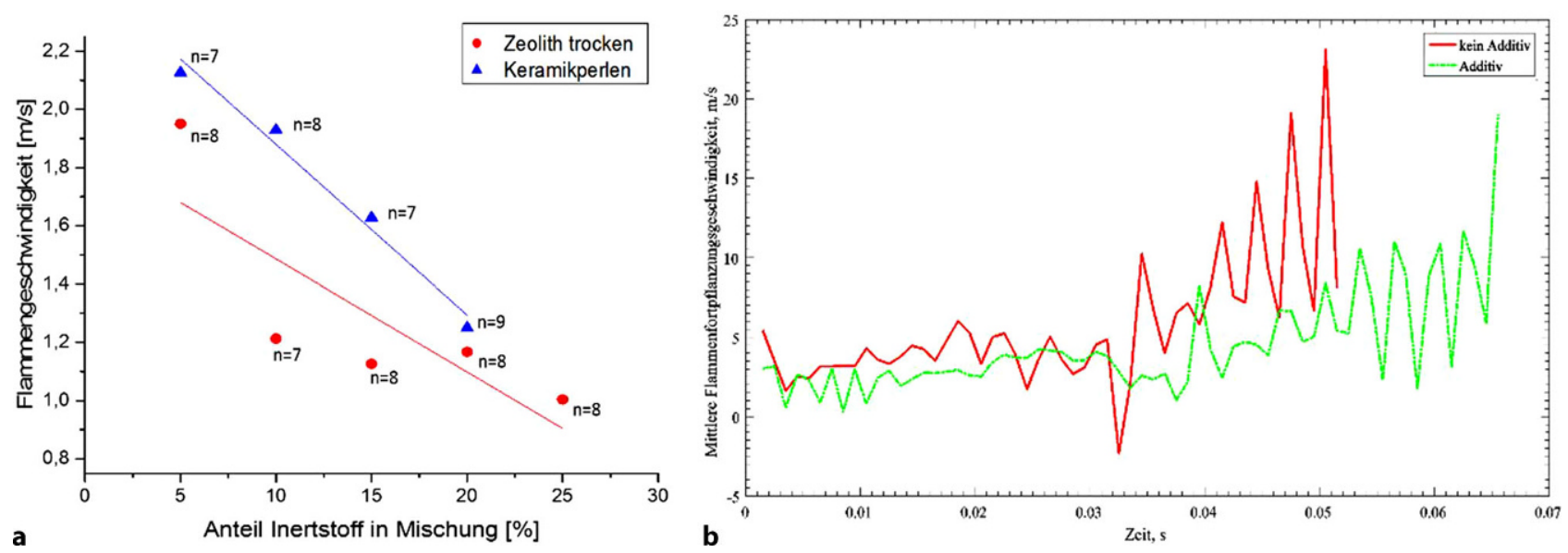

Abb. 3: Versuchsergebnisse Flammengeschwindigkeit (a) und berechnete Flammengeschwindigkeit mittels CFD-Code (b) [11]

einer belichteten Ebene aufgenommen und das Geschwindigkeitsfeld ausgewertet.

\section{Wirkung von Inertstoffen auf die Flammengeschwindigkeit}

Kann die Bildung einer explosionsfähigen Atmosphäre im Betrieb nicht verhindert werden bzw. wenn die Vermeidung von Zündquellen nicht möglich ist, werden Inertmaterialien angewendet, um die ablaufende Kettenreaktion der Verbrennung zu unterbinden. Systeme zur Explosionsunterdrückung werden stationär an der Industrieanlage verbaut und basieren auf der Eindüsung von Trockenchemikalien im Anlassfall. Der Inertisierung durch Inertstoffe liegt neben der thermischen Extinktion (Kühleffekt der Reaktionsgase durch Schmelzen und teilweises Verdampfen der Bestandteile der Partikel) die Reaktion der freien Radikale an der Oberfläche aufgrund deren Beschaffenheit zu Grunde [8]. Dieses Inertisierungsverhalten wurde in Versuchsreihen untersucht. Um die thermische Extinktion bewusst auszuschalten, wurden die Versuche mit Inertstäuben und nicht mit Trockenchemikalien durchgeführt. Das Partikel agiert als Energiesenke und reagiert selbst nicht, stellt aber seine Oberfläche für den dabei wirkenden Wandeffekt bereit. Aufgrund der schnell ablaufenden Oxidationsreaktion kann nur ein kleiner Teil der zur Verfügung stehenden Oberfläche des Inertpartikels wirksam werden. Die Versuchsreihen wurden mit zwei Inertstäuben durchgeführt: Einerseits Keramikperlen, welche durch die ebenmäßige Oberfläche eine sehr geringe spezifische Oberfläche aufweisen, andererseits das kristalline Mineral Zeolith, welches natürlich gewachsene Kanäle und Poren in seiner Oberflächenstruktur aufweist und so eine hohe spezifische Oberfläche besitzt. Als brennbarer Staub wurde Lykopodium verwendet. Charakteristisch für den Sporen des Bärlappgewächses sind sein monodisperses Verhalten und die einheitliche Partikelgröße. Lykopodium besitzt einen netzartigen Aufbau, in dem Ölsäure eingebaut ist, wodurch es leicht entzündbar und schlagartig abbrennt [9]. Der Einfluss der Inertpartikel auf die Flammengeschwindigkeit des verbrennenden Staub/Luft-Gemisches kann
Abb. 3 entnommen werden. Je mehr Inertpartikel in der Inertstoff/Brennstoff/Luft-Mischung vorhanden sind, desto höher ist die zur Verfügung stehende spezifische Oberfläche, und desto langsamer ist die Geschwindigkeit der Flamme, die sich gleichmäßig fortpflanzt. Die Flammengeschwindigkeit sinkt linear ab und strebt gegen jenen Abszissenwert, der den Anteil an Inertstaub in der Mischung angibt, in welcher sich die Verbrennung im gewählten Versuchsaufbau nicht mehr selbstständig fortpflanzen kann [10].

\section{Simulation}

Um die Ergebnisse aus den Laborversuchen noch genauer untersuchen zu können, wurde eine mathematische Modellierung durchgeführt. Dadurch wird die Möglichkeit geschaffen, Größen, auch praktisch nicht messbare, im Modell sowohl räumlich als auch zeitlich sehr fein aufzulösen. Die Computational Fluid Dynamics (CFD)-Simulationen wurden in der open source Software OpenFOAM berechnet. Dabei wurde die Gasphase als Kontinuum betrachtet, für welches die Erhaltungsgleichungen für Masse, Energie, Impuls und Stoffspezies in jeder Gitterzelle gelöst werden. Die Partikel im Fluid wurden mittels Euler-Lagrange Ansatz modelliert und als disperse Phase erfasst, deren Bewegungen mittels Kräftebilanz je Partikel einzeln berechnet werden. Für die Modellierung der Staubexplosion wurde der zur Verfügung gestellte Solver coalChemistryFOAM leicht modifiziert und als Turbulenzmodell das $k-\varepsilon-M o d e l l$ eingeführt. Die Ergebnisse der durchgeführten Simulation sind Abb. $3 \mathrm{~b}$ und 4 zu entnehmen. Die eingesetzten Inertpartikel weisen in Bezug auf ihre Energieaufnahmekapazität Schwächen auf. Zwar sind sie laut den Simulationen in der Lage, die gefährliche Beschleunigungsphase der Explosion stark hinauszuzögern, vollständig unterdrücken konnten sie diese aber nicht [11]. 
Abb. 4: Berechnete Flammenausbreitung im Explosions$\operatorname{rohr}[10]$
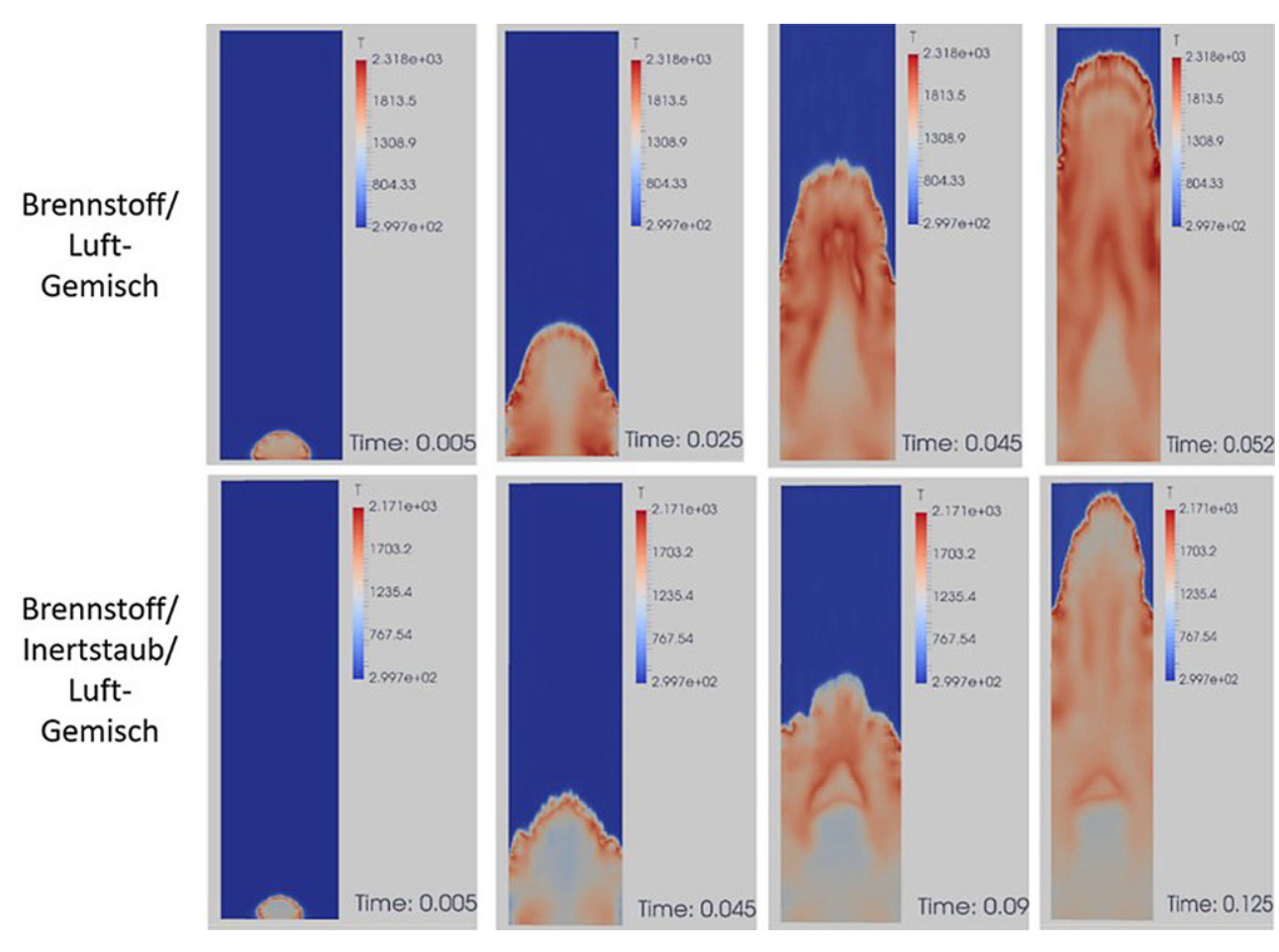

\section{Ausblick}

Durch die Weiterentwicklung von CFD-Berechnungsmodellen durch Realversuche wird es möglich, Sicherheitseinrichtungen für Industrieanlagen anhand der im Labor erhaltenen Kenngrößen zu simulieren und zu berechnen und so an die in der Praxis vorliegenden Bedingungen angepasste Sicherheitseinrichtungen auslegen zu können. Der Lehrstuhl für Thermoprozesstechnik hat hierzu eine zu Normapparaturen alternative Versuchsanlage zur Untersuchung des Explosionsverhaltens von Staub/Luft-Gemischen mit einer Fachfirma entwickelt. In dieser kann der Flammenfortschritt unter möglichst niedrigen Turbulenzbedingungen und unter niedrigem Druckanstieg untersucht werden. Des Weiteren können Informationen über die Flammenausbreitung und das Zündverhalten von (Staub-)Explosionen im Realmaßstab erhalten werden. Die erhaltenen Ergebnisse dienen der Verbesserung von mathematischen Modellen und Berechnungsverfahren, z.B. für Schlagwetterexplosionen oder Explosionen in Behältern. Weitere Untersuchungen sowie Versuche im Großmaßstab sind essentiell für die Validierung der bis dato erhaltenen Ergebnisse und die Weiterentwicklung der Berechnungsmodelle. Hierzu sind weitere Forschungsprojekte in diesem Bereich geplant.

Funding. Open access funding provided by Montanuniversität Leoben.

Open Access Dieser Artikel wird unter der Creative Commons Namensnennung 4.0 International Lizenz veröffentlicht, welche die Nutzung, Vervielfältigung, Bearbeitung, Verbreitung und Wiedergabe in jeglichem Medium und Format erlaubt, sofern Sie den/die ursprünglichen Autor(en) und die Quelle ordnungsgemäß nennen, einen Link zur Creative Commons Lizenz beifügen und angeben, ob Änderungen vorgenommen wurden.
Die in diesem Artikel enthaltenen Bilder und sonstiges Drittmaterial unterliegen ebenfalls der genannten Creative Commons Lizenz, sofern sich aus der Abbildungslegende nichts anderes ergibt. Sofern das betreffende Material nicht unter der genannten Creative Commons Lizenz steht und die betreffende Handlung nicht nach gesetzlichen Vorschriften erlaubt ist, ist für die oben aufgeführten Weiterverwendungen des Materials die Einwilligung des jeweiligen Rechteinhabers einzuholen.

Weitere Details zur Lizenz entnehmen Sie bitte der Lizenzinformation auf http://creativecommons.org/licenses/by/4.0/deed.de.

\section{Literatur}

1. Steen, H.: Handbuch des Explosionsschutzes, Weinheim: WileyVCH, 2009

2. Spijker, C.; Raupenstrauch, H.; Kern, H.; Held, K.: Modeling Dust Explosions, in Proceedings AIChE annual meeting, USA, 2013

3. Krause, U.: Wärme- und Stofftransportvorgänge bei sicherheitstechnischen Untersuchungsverfahren für Staubbrände und Staubexplosionen, Aachen: Shaker Verlag, 2002

4. Kern, H.: Explosible Dust/Air Mixtures. Investigations on flame propagation under non atmospheric conditions, Dissertation, Montanuniversität (Lehrstuhl für Thermorpozestechnik), Leoben, 2013

5. Crowl, D. A.: Understanding explosions, New York: Centre for Chemical Process Safety of the American Institute of Chemical Engineers, 2003

6. Leisenheimer, B.; Leuckel, W.: Schnelle deflagrative, laminare und turbulente $\mathrm{CH} 4 /$ Luft-Flammenfront-Ausbreitung in geschlossenen sphärischen Explosionsbehältern, Chemie Ingenieur Technik, 65 (1993), S. 749-751

7. Wieser, G. J.; Pollhammer, W.; Kern, H.: Einflüsse der Temperatur und der Turbulenz auf die Flammenausbreitung in Staub/Luft-Gemischen unter reduzierten Druckbedingungen, in Proceedings Magdeburger Brand- und Explosionsschutztage, Magdeburg, 2015

8. Dewitte, M.; Vrebosch, J.; van Tiggelen, A.: Inhibition and Extinction of Premixed Flames by Dust Particles, Heterogeneous flame inhibition, 8 (1964), S. 257-266

9. Skold, T.: Selected aspects of turbulence and combustion in 20-litre explosion vessels. Development of experimental apparatus and ex- 
perimental investigation, Master Thesis, University of Bergen (Department of Pysics), Bergen, 2003

10. Hüttenbrenner, K.; Kern, H.: Entzündbarkeit von Staub/Luft-Gemischen bei Verwendung von Inertstäuben mit hoher Porosität, Technische Sicherheit, 8 (2018), S. 25-30

11. Tomasch, S.: Numerische Untersuchung der Wechselwirkung zwischen explosionsfähigen Lykopodium/Luft-Gemischen und inerten partikulären Additiven mittels Euler-Lagrange-Ansatz in OpenFO-

AM, in Proceedings Magdeburger Brand- und Explosionsschutztage, Magdeburg, 2017

Hinweis des Verlags. Der Verlag bleibt in Hinblick auf geografische Zuordnungen und Gebietsbezeichnungen in veröffentlichten Karten und Institutsadressen neutral. 